\title{
Stable Allocations of Risk
}

Citation for published version (APA):

Csóka, P., Herings, P. J. J., \& Kóczy, L. Á. (2009). Stable Allocations of Risk. Games and Economic Behavior, 67(1), 266-277. https://doi.org/10.1016/j.geb.2008.11.001

Document status and date:

Published: 01/01/2009

DOI:

10.1016/j.geb.2008.11.001

Document Version:

Publisher's PDF, also known as Version of record

Document license:
Taverne

\section{Please check the document version of this publication:}

- A submitted manuscript is the version of the article upon submission and before peer-review. There can be important differences between the submitted version and the official published version of record.

People interested in the research are advised to contact the author for the final version of the publication, or visit the DOI to the publisher's website.

- The final author version and the galley proof are versions of the publication after peer review.

- The final published version features the final layout of the paper including the volume, issue and page numbers.

Link to publication

\footnotetext{
General rights rights.

- You may freely distribute the URL identifying the publication in the public portal. please follow below link for the End User Agreement:

www.umlib.nl/taverne-license

Take down policy

If you believe that this document breaches copyright please contact us at:

repository@maastrichtuniversity.nl

providing details and we will investigate your claim.
}

Copyright and moral rights for the publications made accessible in the public portal are retained by the authors and/or other copyright owners and it is a condition of accessing publications that users recognise and abide by the legal requirements associated with these

- Users may download and print one copy of any publication from the public portal for the purpose of private study or research.

- You may not further distribute the material or use it for any profit-making activity or commercial gain

If the publication is distributed under the terms of Article $25 \mathrm{fa}$ of the Dutch Copyright Act, indicated by the "Taverne" license above, 


\title{
Stable allocations of risk
}

\author{
Péter Csóka ${ }^{\mathrm{a}, \mathrm{b}}$, P. Jean-Jacques Herings ${ }^{\mathrm{a}, *}$, László Á. Kóczy ${ }^{\mathrm{a}, \mathrm{c}}$ \\ a Department of Economics, Maastricht University, P.O. Box 616, 6200 MD, Maastricht, The Netherlands \\ b Department of Finance, Corvinus University of Budapest, Hungary \\ ${ }^{\text {c } K e l e t i ~ F a c u l t y ~ o f ~ E c o n o m i c s, ~ B u d a p e s t ~ T e c h, ~ H u n g a r y ~}$
}

A R T I C L E I N F O

Article history:

Received 5 October 2007

Available online 24 November 2008

\section{JEL classification:}

C71

G10

Keywords:

Coherent measures of risk

Risk allocation games

Totally balanced games

Exact games

\begin{abstract}
A B S T R A C T
The measurement and the allocation of risk are fundamental problems of portfolio management. Coherent measures of risk provide an axiomatic approach to the former problem. In an environment given by a coherent measure of risk and the various portfolios' realization vectors, risk allocation games aim at solving the second problem: How to distribute the diversification benefits of the various portfolios? Understanding these cooperative games helps us to find stable, efficient, and fair allocations of risk.

We show that the class of risk allocation and totally balanced games coincide, hence a stable allocation of risk is always possible. When the aggregate portfolio is riskless, the class of risk allocation games coincides with the class of exact games. As in exact games any subcoalition may be subject to marginalization even in core allocations, our result further emphasizes the responsibility involved in allocating risk.
\end{abstract}

(c) 2008 Elsevier Inc. All rights reserved.

\section{Introduction}

The value of an investment portfolio is subject to all kinds of uncertain events. Firms, banks, or insurance companies (to which we refer by the term portfolios) face risk and regulators may require them to hold cash reserves as a cushion against default-this rather unfavorable state of nature-with the precise amounts determined by a measure of risk. A measure of risk thereby specifies the minimal amount of cash the regulated agent has to add to his portfolio for his risk to be acceptable to the regulator.

The literature knows of numerous possible ways to measure risk; lately interest shifted to coherent measures of risk (Artzner et al., 1999) defined by four axioms: monotonicity, subadditivity, positive homogeneity, and translation invariance. These axioms have been shown to be compatible with a natural general equilibrium approach to measure risk (Csóka et al., 2007b).

Of these axioms, subadditivity expresses that the risk of an aggregate portfolio should not exceed the total risk of the individual subportfolios. In particular, the risk of a firm is less than the sum of the risks of the constituents of the firm. Risk allocation then addresses the distribution of the diversification benefits; risk allocation games (Denault, 2001) are transferable utility games defined to this purpose.

A risk allocation game assigns to each coalition of portfolios the risk involved in the aggregate portfolio of the coalition. An allocation shows how to share the risk of the aggregate portfolio of the grand coalition among the individual portfolios,

\footnotetext{
We are grateful to two anonymous referees and conference participants in Warwick, Kos, Madrid, and Budapest for helpful comments. P.J.J. Herings would like to thank the Netherlands Organisation for Scientific Research (NWO) for financial support. L.Á. Kóczy thanks funding by the EU under the Marie Curie Intra-European Fellowship MEIF-CT-2004-011537.

* Corresponding author.

E-mail addresses: Peter.Csoka@uni-corvinus.hu (P. Csóka), P.Herings@algec.unimaas.nl (P.J.J. Herings), Koczy.Laszlo@kgk.bmf.hu (L.Á. Kóczy).
} 
which has of course consequences on the cash reserves to be held. The allocation makes clear what part of the risk of a firm should be attributed to each of its constituents. A natural question that arises is whether there are stable allocations of risk, allocations of risk that no coalition can object to, that is whether the core of the risk allocation game is non-empty.

We separate the risk environment specifying the individual portfolios' realization vectors of discrete random variables and a coherent measure of risk, a real valued function on the realization vectors, from the derived cooperative game that we call risk allocation game.

A totally balanced game is a cooperative game having a non-empty core in all of its subgames. Totally balanced games arise from a wide range of applications. They coincide with market games (Shapley and Shubik, 1969); also with a special case of market games with a continuum of indivisible commodities: cooperation in fair division (Legut, 1990); they are equivalent to a class of maximum flow problems (Kalai and Zemel, 1982a); and also to permutation games of less than four players (Tijs et al., 1984). Moreover, totally balanced games are generated by linear production games (Owen, 1975), generalized network problems (Kalai and Zemel, 1982b), and controlled mathematical programming problems (Dubey and Shapley, 1984).

We show that the class of risk allocation games coincides with the class of totally balanced games, that is all risk allocation games are totally balanced and all totally balanced games can be generated by a risk allocation game with a properly specified risk environment. This result ensures that a regulator can always allocate risk in a stable way. No matter how the risk environment changes, there is always a core element.

We next provide a linear program such that its optimal objective value can be used to determine whether a given cooperative game is a risk allocation game or not. If the game is a risk allocation game, then an optimal solution to the linear program yields a risk environment that generates the game. We then show how to use the linear program to characterize all risk environments that generate a given totally balanced game.

At last, we focus on games where only the distribution of values is uncertain, while the value of the aggregate portfolio is constant over all states of nature. This case is relevant for situations where the risk of the aggregate portfolio is low compared to the risk involved in the individual portfolios. We show that the class of risk allocation games with no aggregate uncertainty coincides with the class of exact games (Schmeidler, 1972). As evidenced by the previous paragraphs, there are many applications giving rise to the class of totally balanced games. There are few applications which lead to exact games. The only example we know of is Calleja et al. (2005), who show that the class of multi-issue allocation games coincides with the class of nonnegative exact games.

The fact that each risk allocation game is exact implies that for each coalition there is a core element such that the coalition only gets its stand-alone value. This means that in the case of no aggregate uncertainty, this coalition does not necessarily benefit from the diversification opportunities offered by the aggregate portfolio. As a consequence, the regulator has a high level of discretion in allocating the risk to the individual portfolios.

The structure of the paper is as follows. First we introduce coherent measures of risk, transferable utility games, and risk allocation games. In Section 3 we prove that the class of risk allocation games coincides with the class of totally balanced games and investigate our constructive proof by linear programming. In Section 4 we show that the class of risk allocation games with no aggregate uncertainty coincides with the class of exact games. In Section 5 we conclude.

\section{Preliminaries}

\subsection{Coherent measures of risk}

Consider the set $\mathbb{R}^{S}$ of realization vectors, where $S$ denotes the number of states of nature. State of nature $s$ occurs with probability $p_{s}>0$ and $\sum_{s=1}^{S} p_{s}=1$. The vector $X \in \mathbb{R}^{S}$ represents a portfolio's possible profit and loss realizations on a common chosen future time horizon, say at $t=1$. The amount $X_{s}$ is the portfolio's payoff in state of nature $s$. Negative values of $X_{S}$ correspond to losses. The inequality $Y \geqslant X$ means that $Y_{s} \geqslant X_{S}$ for all $s=1, \ldots, S$.

A measure of risk is a function $\rho: \mathbb{R}^{S} \rightarrow \mathbb{R}$ measuring the risk of a portfolio from the perspective of the present $(t=0)$. It is the minimal amount of cash the regulated agent has to add to his portfolio, and to invest in a reference instrument today, such that it ensures that the risk involved in the portfolio is acceptable to the regulator. We assume that the reference instrument has payoff 1 in each state of nature at $t=1$, thus its realization vector is $1^{S}=(1, \ldots, 1)^{\top}$. The reference instrument is riskless in the "classical sense," having no uncertainty in its payoffs. It is most natural to think of it as a zero coupon bond. The price of the reference instrument is denoted by $\delta \in \mathbb{R}_{+}$, where $\mathbb{R}_{+}=[0, \infty)$. We adjust the definition of coherent measures of risk to the discrete case with realization vectors as follows.

Definition 2.1. A function $\rho: \mathbb{R}^{S} \rightarrow \mathbb{R}$ is called a coherent measure of risk (Artzner et al., 1999) if it satisfies the following axioms:

1. Monotonicity: for all $X, Y \in \mathbb{R}^{S}$ such that $Y \geqslant X$, we have $\rho(Y) \leqslant \rho(X)$.

2. Subadditivity: for all $X, Y \in \mathbb{R}^{S}$, we have $\rho(X+Y) \leqslant \rho(X)+\rho(Y)$.

3. Positive homogeneity: for all $X \in \mathbb{R}^{S}$ and $h \in \mathbb{R}_{+}$, we have $\rho(h X)=h \rho(X)$.

4. Translation invariance: for all $X \in \mathbb{R}^{S}$ and $a \in \mathbb{R}$, we have $\rho\left(X+a 1^{S}\right)=\rho(X)-\delta a$. 
Acerbi (2002) treats a subclass of coherent measures of risk: spectral measures of risk. The definition of spectral measures of risk with equiprobable outcomes, i.e. $p_{1}=\cdots=p_{S}=1 / S$, is as follows.

Let us introduce the ordered statistics $X_{S: S}$ given by the ordered values of the $S$-tuple $X_{1}, \ldots, X_{S}$, that is $\left\{X_{1: S}, \ldots, X_{S: S}\right\}=\left\{X_{1}, \ldots, X_{S}\right\}$ and $X_{1: S} \leqslant X_{2: S} \leqslant \cdots \leqslant X_{S: S}$.

Definition 2.2. Let the outcomes be equiprobable. Consider a vector $\phi \in \mathbb{R}^{S}$. The measure of risk $M_{\phi}: \mathbb{R}^{S} \rightarrow \mathbb{R}$ defined by

$$
M_{\phi}(X)=-\delta \sum_{S=1}^{S} \phi_{s} X_{s: S}
$$

is a spectral measure of risk if $\phi \in \mathbb{R}^{S}$ satisfies the conditions:

1. Nonnegativity: $\phi_{s} \geqslant 0$ for all $s=1, \ldots, S$.

2. Normalization: $\sum_{s=1}^{S} \phi_{s}=1$.

3. Monotonicity: $\phi_{s}$ is non-increasing, that is $\phi_{s_{1}} \geqslant \phi_{s_{2}}$ if $s_{1}<s_{2}$ and $s_{1}, s_{2} \in\{1, \ldots, S\}$.

Spectral measures of risk are discounted weighted average losses, with non-increasing weights, with the highest weight on the worst outcome. The weight vector $\phi$ is the so-called risk spectrum, the "attitude" toward risk. An important example of a spectral measure of risk is the $k$-expected shortfall.

Definition 2.3. Let the outcomes be equiprobable and let $k \in\{1, \ldots, S\}$. The $k$-expected shortfall of the realization vector $X$ is defined by

$$
\mathrm{ES}_{k}(X)=-\delta \sum_{s=1}^{k} \frac{1}{k} X_{s: S} .
$$

The $k$-expected shortfall is the discounted average of the worst $k$ outcomes. For a detailed discussion see Acerbi and Tasche (2002).

\subsection{Transferable utility games}

Let $N=\{1, \ldots, n\}$ denote a finite set of players. A value function $v: 2^{N} \rightarrow \mathbb{R}$ with $v(\{\emptyset\})=0$ gives rise to a cooperative game with transferable utility (game, for short) $(N, v)$. Let $\Gamma$ denote the set of games with $n$ players. An allocation is a vector $x \in \mathbb{R}^{n}$, where $x_{i}$ is the payoff of player $i \in N$. An allocation $x$ yields payoff $x(C)=\sum_{i \in C} x_{i}$ to a coalition $C \in 2^{N}$. An allocation $x \in \mathbb{R}^{n}$ is called efficient, if $x(N)=v(N)$; individually rational, if $x_{i} \geqslant v(\{i\})$ for all $i \in N$, and coalitionally rational if $x(C) \geqslant v(C)$ for all $C \in 2^{N}$. The core is the set of efficient and coalitionally rational allocations.

For each $C \in 2^{N}$ let $a(C) \in \mathbb{R}^{n}$ be the membership vector, $a_{i}(C)=1$ for $i \in C$ and $a_{i}(C)=0$ otherwise.

Definition 2.4. A balanced vector of weights is a vector $\left(\lambda^{C}\right)_{C \in 2^{N}} \in \mathbb{R}_{+}^{2^{N}}$ such that $\sum_{C \in 2^{N}} \lambda^{C} a(C)=a(N)$. A game $(N, v)$ is balanced if $\sum_{C \in 2^{N}} \lambda^{C} v(C) \leqslant v(N)$ for all balanced vectors of weights.

A well-known interpretation of balancedness is that the players can distribute one unit of working time to any coalition and if each coalition is active during a fraction $\lambda^{C}$ of a unit of time then the players cannot generate more value than $v(N)$, the value of the grand coalition. Balancedness is a necessary and sufficient condition for non-emptiness of the core in a transferable utility game (Bondareva, 1963; Shapley, 1967). See Predtetchinski and Herings (2004) for an extension of the concept of balancedness to be necessary and sufficient for non-emptiness of the core in non-transferable utility games.

For a game $(N, v)$ and a coalition $C \in 2^{N}$, a subgame $\left(C, v^{C}\right)$ is obtained by restricting $v$ to subsets of $C$.

Definition 2.5. A game $(N, v)$ is totally balanced if for every $D \in 2^{N}$ its subgame $\left(D, v^{D}\right)$ is balanced, that is, if for all $D \in 2^{N}$ and for all vectors $\left(\lambda^{C}\right)_{C \in 2^{D}} \in \mathbb{R}_{+}^{2^{D}}$ satisfying $\sum_{C \in 2^{D}} \lambda^{C} a(C)=a(D)$, we have $\sum_{C \in 2^{D}} \lambda^{C} v(C) \leqslant v(D)$.

In a totally balanced game every subgame has a non-empty core. Let $\Gamma_{\mathrm{tb}}$ denote the family of totally balanced games with $n$ players. An interesting subclass of totally balanced games is the class of exact games (Shapley, 1971; Schmeidler, 1972).

Definition 2.6. A game $(N, v)$ is exact if for each $C \in 2^{N}$ there exists a core allocation $x$ such that $x(C)=v(C)$.

Schmeidler (1972) characterizes exact games as follows (see also Derks and Reijnierse, 1998, Theorem 7). 
Theorem 2.7. (See Schmeidler, 1972.) A game $(N, v) \in \Gamma$ is exact if and only if for every $D \in \mathcal{N} \backslash\{N\}$, for each vector $\left(\gamma^{C}\right)_{C \in \mathcal{N}}$ such that $\gamma^{C} \in \mathbb{R}_{+}$and $\sum_{C \in \mathcal{N} \backslash\{N\}} \gamma^{C} a(C)=a(D)+\gamma^{N} a(N)$ we have $\sum_{C \in \mathcal{N} \backslash\{N\}} \gamma^{C} v(C) \leqslant v(D)+\gamma^{N} v(N)$.

Let $\Gamma_{\mathrm{e}}$ denote the family of exact games with $n$ players. Convex games (Shapley, 1971) are a subset of exact games.

Definition 2.8. A game $(N, v)$ is convex if for all $C, D \in 2^{N}$ we have that $v(C)+v(D) \leqslant v(C \cup D)+v(C \cap D)$.

Let $\Gamma_{\mathrm{c}}$ denote the family of convex games with $n$ players. We have that $\Gamma \supseteq \Gamma_{\mathrm{tb}} \supseteq \Gamma_{\mathrm{e}} \supseteq \Gamma_{\mathrm{c}}$.

\subsection{Risk allocation games}

Denault (2001) introduces risk capital allocation problems: Suppose a firm has $n$ constituents and the matrix of their realization vectors is given by $X \in \mathbb{R}^{S \times n}$. ${ }^{1}$ The question is how the risk of the firm as measured by a coherent measure of risk has to be allocated to its constituents in a stable way, meaning that the risk allocated to a coalition of constituents does not exceed the risk of the coalition's aggregate portfolio.

Let $X_{. i}$ denote the $i$ th column of $X$, the realization vector of portfolio $i$. Let $X_{s}$. denote the row of $X$ corresponding to state of nature $s, X_{s, i}$ its element at row $s$ and column $i$, and $\left(X_{s, i}\right)_{i \in D}$ the row vector corresponding to state of nature $s$ with elements $i \in D$. For a coalition of portfolios $C \in 2^{N}$, let $X(C)=\sum_{i \in C} X_{. i}$ and $X_{S}(C)=\sum_{i \in C} X_{s, i}$.

Denault (2001) assumes that the $n$th portfolio equals $b \in \mathbb{R}$ units of the reference instrument: $X \cdot n=b 1^{S}$. We will consider the slightly more general setting where $X_{. n}$ can be any portfolio. Moreover, we make a distinction between the risk environment and the induced game.

Definition 2.9. A risk environment is a tuple $(N, S, p, X, \rho)$, where $N$ is the set of portfolios, $S$ indicates the number of states of nature, $p=\left(p_{1}, \ldots, p_{S}\right)$ is the vector of realization probabilities of the various states, $X$ is the matrix of realization vectors, and $\rho$ is a coherent measure of risk.

Definition 2.10. Given a risk environment $(N, S, p, X, \rho)$ a risk allocation game is a game $(N, v)$, where the value function $v: 2^{N} \rightarrow \mathbb{R}$ is defined by

$$
v(C)=-\rho(X(C)) \text { for all } C \in 2^{N} .
$$

A risk allocation game with $n$ players is induced by the number of states of nature, their probability of occurrence, $n$ realization vectors and a coherent measure of risk. Let $\Gamma_{\mathrm{r}}$ denote the family of risk allocation games with $n$ players. In such a game, according to Eq. (3), the larger the risk of any subset of portfolios, the lower its value.

If the rows of a matrix of realization vectors sum up to the same number, then there is no aggregate uncertainty. Formally:

Definition 2.11. A matrix of realization vectors $X \in \mathbb{R}^{S \times n}$ has no aggregate uncertainty if there exists a number $\alpha \in \mathbb{R}$ such that $X(N)=\alpha 1^{S}$.

Let $\Gamma_{\text {rnau }}$ denote the family of risk allocation games with $n$ players with no aggregate uncertainty. Obviously, $\Gamma_{\text {rnau }} \subseteq \Gamma_{\mathrm{r}}$. We first study risk allocation games in general, then with no aggregate uncertainty.

\section{Total balancedness}

\subsection{Risk allocation games and totally balanced games}

Denault (2001, Theorem 4) shows that the family of risk capital allocation problems is balanced. As a subgame of a risk allocation game is also a risk allocation game, we can adjust his proof to show that risk allocation games are totally balanced.

Proposition 3.1. All games $(N, v) \in \Gamma_{\mathrm{r}}$ are totally balanced, $\Gamma_{\mathrm{r}} \subseteq \Gamma_{\mathrm{tb}}$.

Proof. Consider a risk environment $(N, S, p, X, \rho)$ inducing the game $(N, v)$. We show that for any $D \in 2^{N}$, the subgame $\left(D, v^{D}\right)$ is balanced. Take any $\left(\lambda^{C}\right)_{C \in 2^{D}} \in \mathbb{R}_{+}^{2^{D}}$ such that $\sum_{C \in 2^{D}} \lambda^{C} a(C)=a(D)$. Then by Eq. (3) and the positive homogeneity and subadditivity of $\rho$ we have that

\footnotetext{
1 Denault (2001) uses continuously distributed random variables. We adjust his setting to the more tractable setup with discrete random variables, resulting in realization vectors.
} 


$$
\begin{aligned}
\sum_{C \in 2^{D}} \lambda^{C} v^{D}(C) & =-\sum_{C \in 2^{D}} \rho\left(\lambda^{C} X(C)\right) \leqslant-\rho\left(\sum_{C \in 2^{D}}\left(\sum_{i \in C} \lambda^{C} X_{\cdot i}\right)\right) \\
& =-\rho\left(\sum_{i \in D}\left(\sum_{C \in 2^{D}, C \ni i} \lambda^{C} X_{. i}\right)\right)=-\rho\left(\sum_{i \in D} X_{. i}\right)=-\rho(X(D)) \\
& =v^{D}(D),
\end{aligned}
$$

where the last line follows from rearranging the summation and using the fact that we have a balanced vector of weights. Thus $\left(D, v^{D}\right)$ is balanced.

Not only is it true that all risk allocation games are totally balanced, but also any totally balanced game can be generated by a risk allocation game. We illustrate Proposition 3.2 and its proof by Example 3.3.

Proposition 3.2. Each game $(N, v) \in \Gamma_{\mathrm{tb}}$ is induced by some risk environment $(N, S, p, X, \rho)$, so $\Gamma_{\mathrm{tb}} \subseteq \Gamma_{\mathrm{r}}$.

Proof. Take any game $(N, v) \in \Gamma_{\mathrm{tb}}$. The zero-normalized value function $v_{0}$ corresponding to $v$ is defined by

$$
v_{0}(C)=v(C)-\sum_{i \in C} v(\{i\}), \quad C \in 2^{N} .
$$

It is well known that $\left(N, v_{0}\right) \in \Gamma_{\mathrm{tb}}$. Using the singletons with weights 1 it follows from the total balancedness of $v_{0}$ that for any $C \in 2^{N}$

$$
0=\sum_{i \in C} v_{0}(\{i\}) \leqslant v_{0}(C)
$$

Moreover, any $C \in 2^{N}$ partitions $N$ into $C$ and $N \backslash C$, and using weights 1 on $C$ and $N \backslash C$ leads to

$$
v_{0}(C)+v_{0}(N \backslash C) \leqslant v_{0}(N) .
$$

Using Eqs. (5) and (6) we obtain that for any $C \in 2^{N}$

$$
0 \leqslant v_{0}(C) \leqslant v_{0}(N) .
$$

The remainder of the proof is constructive. We specify the risk environment $\left(N, S, p, X^{0}, \rho\right)$ as follows. We introduce a state of nature for all non-empty coalitions of $N$, so $S=2^{n}-1$. We label states of nature by $C, D \in 2^{N} \backslash\{\emptyset\}$. We consider equiprobably outcomes, $p_{1}=\cdots=p_{S}=1 / S$, and we let $\rho$ be the risk measure equal to the 1-expected shortfall (Definition 2.3) with $\delta=1$. For each state of nature $C \in 2^{N} \backslash\{\emptyset\}$, let the row vector $X_{C \text {. }}^{0}$ be such that

$$
\begin{aligned}
& \left(X_{C, i}^{0}\right)_{i \in C} \text { belongs to the core of }\left(C, v_{0}^{C}\right), \\
& X_{C, i}^{0}=v_{0}(N), \quad i \in N \backslash C .
\end{aligned}
$$

The risk environment $\left(N, S, p, X^{0}, \rho\right)$ induces the game $\left(N, \bar{v}_{0}\right)$. We will show that $\bar{v}_{0}=v_{0}$.

By the definition of 1-expected shortfall, we have

$$
\bar{v}_{0}(C)=-\rho\left(X^{0}(C)\right)=\min _{D \in 2^{N} \backslash\{\emptyset\}} X_{D}^{0}(C), \quad C \in 2^{N} .
$$

The definition of a subgame, Eq. (8), and the efficiency of a core element imply

$$
v_{0}^{C}(C)=v_{0}(C)=X_{C}^{0}(C), \quad C \in 2^{N} \backslash\{\emptyset\} .
$$

We show next that

$$
X_{C}^{0}(C) \leqslant X_{D}^{0}(C), \quad C, D \in 2^{N} \backslash\{\emptyset\} .
$$

Indeed, if $D \supseteq C$ then inequality (12) follows from (8) as we have for a core element $\left(X_{D, i}^{0}\right)_{i \in D}$ in subgame $\left(D, v_{D}^{0}\right)$ that

$$
X_{C}^{0}(C)=v_{0}(C) \leqslant X_{D}^{0}(C) .
$$

If $D \nsupseteq C$ then one of the components of $\left(X_{D, i}^{0}\right)_{i \in C}$ is $v_{0}(N)$, and using Eq. (7) inequality (12) follows immediately. Combining Eqs. (11) and (12) with Eq. (10) we obtain that $\bar{v}_{0}=v_{0}$.

By using the matrix of realization vectors $X$ defined by $X_{. i}=X_{. i}^{0}+v(\{i\}) 1^{S}, i \in N$, we obtain a risk environment that induces the game $(N, v)$. 
Table 1

A totally balanced game and its zero-normalized game.

\begin{tabular}{lcc}
\hline$C$ & $v(C)$ & $v_{0}(C)$ \\
$\{1\}$ & -10 & 0 \\
$\{2\}$ & 3 & 0 \\
$\{3\}$ & -2 & 0 \\
$\{1,2\}$ & -4 & 3 \\
$\{1,3\}$ & -6 & 2 \\
$\{1,3\}$ & -1 & 1 \\
\hline
\end{tabular}

Table 2

Payoff matrices for the zero normalized and the original games.

\begin{tabular}{|c|c|c|c|c|c|c|}
\hline$S$ & $X_{\cdot 1}^{0}$ & $X_{.2}^{0}$ & $X_{.3}^{0}$ & $X_{.1}$ & $X_{\cdot 2}$ & $X_{.3}$ \\
\hline$\{1\}$ & 0 & 8 & 8 & -10 & 11 & 6 \\
\hline$\{2\}$ & 8 & 0 & 8 & -2 & 3 & 6 \\
\hline$\{3\}$ & 8 & 8 & 0 & -2 & 11 & -2 \\
\hline$\{1,2\}$ & 1 & 2 & 8 & -9 & 5 & 6 \\
\hline$\{1,3\}$ & 2 & 8 & 4 & -8 & 11 & 2 \\
\hline$\{2,3\}$ & 8 & 1 & 0 & -2 & 4 & -2 \\
\hline$\{1,2,3\}$ & 2 & 1 & 5 & -8 & 4 & 3 \\
\hline
\end{tabular}

Example 3.3. We illustrate the construction used in the proof of Proposition 3.2 in an example with 3 players. Table 1 presents the value function $v$ of a totally balanced game, as well as the zero-normalized value function $v_{0}$ corresponding to $v$. Note that inequality (7) is satisfied by $v_{0}$.

In Table 2 we have specified the matrix of realization vectors $X^{0}$ according to requirements (8) and (9). For instance, for $C=\{1,2\}$ we have that $\left(X_{\{1,2\}, 1}^{0}, X_{\{1,2\}, 2}^{0}\right)=(1,2)$ is a point in the core of the subgame with players 1 and 2 , and $X_{\{1,2\}, 3}^{0}=8=v_{0}(N)$.

It is easy to check that the risk environment specified by $X^{0}$ and the risk measure equal to the 1-expected shortfall with $\delta=1$ generate $v_{0}$.

To generate the value function $v$, we transform $X^{0}$ into $X$ by specifying $X_{. i}=X_{. i}^{0}+v(\{i\}) 1^{S}$ for all $i \in N$. The risk environment corresponding to $X$ and the risk measure equal to the 1-expected shortfall with $\delta=1$ can be verified to induce the game $(N, v)$.

Note that in our constructive proof the statement of Proposition 3.2 is strengthened in the sense that the family of games induced by risk environments with $S \leqslant 2^{n}-1$ and the risk measure equal to the 1-expected shortfall with $\delta=1$ coincides with the family of totally balanced games with $n$ players, that is any totally balanced game can be generated by a properly specified risk environment with the 1-expected shortfall and $2^{n}-1$ states of nature. From Propositions 3.1 and 3.2 we derive the following theorem.

Theorem 3.4. The class of risk allocation games coincides with the class of totally balanced games, $\Gamma_{\mathrm{r}}=\Gamma_{\mathrm{tb}}$.

Kalai and Zemel (1982b) use a similar construction to show that a game is totally balanced if and only if it is the minimum game of a finite collection of additive games. A game $(N, v)$ is called additive if there exists a set of real numbers $b_{1}, \ldots, b_{n}$ such that for every $C \in 2^{N}, v(C)=\sum_{i \in C} b_{i}$. For a finite collection of games $\left\{v_{t}\right\}_{t \in T}$ the minimum game is defined by $\left(\min v_{t}\right)(C)=\min _{t \in T} v_{t}(C)$. It is easy to see that the totally balanced game $v$ in Table 1 is equal to the minimum game of the collection of additive games generated by $X_{C}, C \in 2^{N} \backslash\{\varnothing\}$, in Table 2 .

\subsection{Linear programming results}

Consider a totally balanced game $(N, v) \in \Gamma_{\mathrm{tb}}$. Throughout the subsection, we choose $S=2^{n}-1, p_{1}=\cdots=p_{S}=1 / S$, and $\rho$ the risk measure equal to 1-expected shortfall with $\delta=1$, just like in Proposition 3.2. Whenever we write $v$ is generated by a matrix of realization vectors $X$ we mean that the risk allocation game induced by the risk environment $(N, S, p, X, \rho)$ equals $(N, v)$.

In the proof of Proposition 3.2 the matrix of realization vectors $X$ generating $v$ was constructed using the core requirement ${ }^{2}$ (8): for every $C \in 2^{N} \backslash\{\emptyset\}$

$$
\left(X_{C, i}\right)_{i \in C} \text { belongs to the core of }\left(C, v^{C}\right) .
$$

\footnotetext{
2 There we had a zero normalized game, but it is easy to see that after renormalizing the core requirement is still satisfied.
} 
The other elements of $X$ were chosen to be sufficiently large.

Let a game $(N, v) \in \Gamma$ be given. We develop a linear programming problem such that the optimal value of the objective function will exceed $\sum_{C \in 2^{N}} v(C)$ if $(N, v) \in \Gamma \backslash \Gamma_{\text {tb }}$ and is equal to $\sum_{C \in 2^{N}} v(C)$ whenever the game is totally balanced. Moreover, in the latter case the matrices derived from the linear program's optimal solutions generate $v$.

To do so, given a matrix $X$ we define the vector $\hat{X} \in \mathbb{R}^{S n}$ by juxtaposing the rows of $X \in \mathbb{R}^{S \times n}$, that is $\hat{X}=$ $\left(X_{1}, X_{2}, \ldots, X_{S} .\right)^{\top} \in \mathbb{R}^{S n}$, and the reverse operation transforms a vector $\hat{X} \in \mathbb{R}^{S n}$ into a matrix $X \in \mathbb{R}^{S \times n}$. We will use the notations $\hat{X}$ and $X$ interchangeably.

Let $0^{n}=(0,0, \ldots, 0) \in \mathbb{R}^{1 \times n}$ be the $n$-dimensional row vector of zeros. For every $C \in 2^{N} \backslash\{\emptyset\}$, we define the matrices

$$
A(C)=\left(\begin{array}{cccc}
a(C)^{\top} & 0^{n} & & 0^{n} \\
0^{n} & a(C)^{\top} & & 0^{n} \\
& & \ddots & \vdots \\
0^{n} & 0^{n} & \cdots & a(C)^{\top}
\end{array}\right) \in \mathbb{R}^{S \times S n}
$$

containing the membership vector $a(C)$ transposed along the "diagonal" and $0^{n}$ otherwise.

A matrix $X \in \mathbb{R}^{S \times n}$ generates $v$ if and only if for every $C \in 2^{N} \backslash\{\varnothing\}$

$$
v(C)=\min _{D \in 2^{N} \backslash\{\emptyset\rceil} X_{D}(C) .
$$

Eq. (16) can be rewritten as

$$
v(C)=\min _{D \in 2^{N}} A_{D \cdot}(C) \hat{X},
$$

where $A_{D}$. (C) denotes the $D$ th row of $A(C)$. It follows from Eq. (17) that $X$ generates $v$ if and only if for every $C \in 2^{N} \backslash\{\emptyset\}$

$$
A(C) \hat{X} \geqslant v(C) 1^{S},
$$

where for each $C$ at least one inequality holds with equality.

We introduce some additional notation. Let

$$
\begin{aligned}
& E=\left(a(\{1\})^{\top}, a(\{2\})^{\top}, \ldots, a(N)^{\top}\right) \in \mathbb{R}^{1 \times S n}, \\
& V=\left(\begin{array}{c}
v(\{1\}) 1^{S} \\
v(\{2\}) 1^{S} \\
\vdots \\
v(N) 1^{S}
\end{array}\right) \in \mathbb{R}^{S^{2}},
\end{aligned}
$$

and

$$
A=\left(\begin{array}{c}
A(\{1\}) \\
A(\{2\}) \\
\vdots \\
A(N)
\end{array}\right) \in \mathbb{R}^{S^{2} \times S n} .
$$

Consider the linear programming problem $\left(\mathrm{P}_{v}\right)$ :

$\left(\mathrm{P}_{v}\right) \min E \hat{X}$

$$
\begin{array}{ll}
\text { s.t. } & A \hat{X} \geqslant V, \\
& \hat{X} \in \mathbb{R}^{S n} .
\end{array}
$$

The objective function of $\left(\mathrm{P}_{v}\right)$ captures the constructive proof of Proposition 3.2, as it is minimizing exactly the sum of those elements of $\hat{X}$ which are used in the core requirement (14). Using Eq. (18) it can be seen that the feasibility constraints in the linear program are the necessary requirements for $v$ to be generated by a feasible solution.

The set of optimal solutions of $\left(\mathrm{P}_{v}\right)$ is non-empty, since $\hat{X}=(k, \ldots, k) \in \mathbb{R}^{S n}$ is a feasible solution, where $k=$ $\max _{C \in 2^{N}} v(C)$ and the set of feasible solutions is bounded from below. We denote the set of optimal solutions of $\left(\mathrm{P}_{v}\right)$ by $\mathcal{X}_{v}^{*}$ and a particular optimal solution by $\hat{X}^{*} \in \mathbb{R}^{S n}$.

Proposition 3.5. Consider a game $(N, v) \in \Gamma$ and an optimal solution $\hat{X}^{*} \in \mathcal{X}_{v}^{*}$ of $\left(\mathrm{P}_{v}\right)$. The optimal value of the objective function $E \hat{X}^{*}$ equals $\sum_{C \in 2^{N}} v(C)$ if and only if $v$ is generated by $X^{*}$. 
Proof. $(\Rightarrow)$ By the feasibility constraints it holds that

$$
A_{D \cdot}(C) \hat{X}^{*} \geqslant v(C), \quad C \in 2^{N} \backslash\{\emptyset\}, D \in 2^{N} \backslash\{\emptyset\} .
$$

Since by assumption $E \hat{X}^{*}=\sum_{C \in 2^{N}} v(C)$, it follows that

$$
A_{C \cdot}(C) \hat{X}^{*}=v(C), \quad C \in 2^{N} \backslash\{\emptyset\} .
$$

It follows by inequality (21) and Eq. (22) that $v$ is generated by $X^{*}$.

$(\Leftarrow)$ We use a proof by contradiction. Suppose $E \hat{X}^{*} \neq \sum_{C \in 2^{N}} v(C)$. By the feasibility constraints it holds that

$$
E \hat{X}^{*}>\sum_{C \in 2^{N}} v(C) .
$$

Note that $\min _{D \in 2^{N} \backslash\{\emptyset\}} X_{D}^{*}(C)$ is attained in row $C$ of $X^{*}$, since otherwise we could decrease the objective function by substituting the row attaining the minimum for row $C$. Combining this with Eq. (23) we obtain that there exists a coalition $C \in 2^{N}$ such that

$$
\min _{D \in 2^{N} \backslash\{\emptyset\}} X_{D}^{*}(C)>v(C),
$$

which implies that $v$ is not generated by $X^{*}$, a contradiction.

Take any matrix of realization vectors $X \in \mathbb{R}^{z \times n}$, where $z$ is a strictly positive integer. Let $Y(X) \in \mathbb{R}^{\left(2^{n}-1\right) \times n}$ denote a matrix in which for all $C \in 2^{N} \backslash\{\emptyset\}$ we have that $Y_{C} .(X)=X_{k}$, where $k \in \arg \min _{k \in\{1,2, \ldots, z\}} X_{k}(C)$. The following proposition claims that the realization matrix $X$ generates $v$ if and only if $\hat{Y}(X)$ is an optimal solution of $\left(\mathrm{P}_{v}\right)$.

Proposition 3.6. Consider a game $(N, v) \in \Gamma_{\mathrm{tb}}$. The matrix of realization vectors $X \in \mathbb{R}^{z \times n}$ generates $v$ if and only if $\hat{Y}(X) \in \mathcal{X}_{v}^{*}$.

Proof. $(\Rightarrow)$ Since $X$ generates $v$, for all $C \in 2^{N} \backslash\{\emptyset\}$ there exists a state of nature $s(C)$ such that

$$
\begin{aligned}
& X_{S(C)}(C)=v(C), \\
& X_{S}(C) \geqslant v(C), \quad s \in\{1, \ldots, S\} .
\end{aligned}
$$

It follows that $\hat{Y}(X)$ is a feasible and optimal solution of $\left(\mathrm{P}_{v}\right)$.

$(\Leftarrow)$ Since the game $(N, v)$ is totally balanced, according to Theorem $3.4 v$ is generated by some matrix of realization vectors. By Proposition 3.5, it follows that $v$ is generated by all elements of $\mathcal{X}_{v}^{*}$, so $Y(X)$ generates $v$, and by construction $X$ generates $v$.

The following result shows that any matrix of realization vectors $X$ that generates $v$ satisfies (14).

Proposition 3.7. Consider a game $(N, v) \in \Gamma_{\mathrm{tb}}$. Any optimal solution of $\left(\mathrm{P}_{v}\right) \hat{X}^{*} \in \mathcal{X}_{v}^{*}$ satisfies the core requirement (14).

Proof. Take any $\hat{X}^{*} \in \mathcal{X}_{v}^{*}$. Since by Proposition 3.2 all totally balanced games can be generated, we know by Proposition 3.5 that $E \hat{X}^{*}=\sum_{C \in 2^{N}} v(C)$. For every $C \in 2^{N} \backslash\{\emptyset\}$, feasibility requires that $A_{C}$. $(C) \hat{X}^{*} \geqslant v(C)$, so

$$
A_{C} \cdot(C) \hat{X}^{*}=v(C) \text {. }
$$

The equalities in (25) together with the feasibility constraints imply that the rows of $X^{*}$ contain core allocations of the respective subgames.

Propositions 3.6 and 3.7 imply that if a game is generated by $X \in \mathbb{R}^{z \times n}$, then $Y(X)$ satisfies the core requirement (14). Thus to generate a given totally balanced game the rows of the matrix of realization vectors can be permutated and some of them can be combined, but essentially the core requirement (14) is satisfied in all of them.

\section{Exactness}

In this section we show that if there is no aggregate uncertainty in a risk environment, then the induced risk allocation game is an exact game, and conversely all exact games can be generated by a properly specified risk environment with no aggregate uncertainty. Proposition 4.1 claims that risk allocation games with no aggregate uncertainty are exact.

Proposition 4.1. All games with $(N, v) \in \Gamma_{\text {rnau }}$ are exact, $\Gamma_{\text {rnau }} \subseteq \Gamma_{\mathrm{e}}$. 
Proof. Consider the risk environment $(N, S, p, X, \rho)$, where $X$ has no aggregate uncertainty. We show that the induced risk allocation game is exact by Theorem 2.7.

Take any $D \in \mathcal{N} \backslash\{N\}$ and any vector $\left(\gamma^{C}\right)_{C \in \mathcal{N}}$ such that $\gamma^{C} \in \mathbb{R}_{+}$and

$$
\sum_{C \in \mathcal{N} \backslash\{N\}} \gamma^{C} a(C)=a(D)+\gamma^{N} a(N) \text {. }
$$

Then, by Eq. (3), the positive homogeneity, and subadditivity of $\rho$ we have that

$$
\begin{aligned}
\sum_{C \in \mathcal{N} \backslash\{N\}} \gamma^{C} v(C) & =-\sum_{C \in \mathcal{N} \backslash\{N\}} \rho\left(\sum_{i \in C} \gamma^{C} X_{\cdot i}\right) \\
& \leqslant-\rho\left(\sum_{C \in \mathcal{N} \backslash\{N\}}\left(\sum_{i \in C} \gamma^{C} X_{\cdot i}\right)\right) \\
& =-\rho\left(\sum_{i \in N}\left(\sum_{C \ni i, C \in \mathcal{N} \backslash\{N\}} \gamma^{C} X_{\cdot i}\right)\right) \\
& =-\rho\left(\sum_{i \in D} X_{\cdot i}+\gamma^{N} \sum_{i \in N} X_{\cdot i}\right),
\end{aligned}
$$

where the last two lines follow from rearranging the summation and using Eq. (26), thus if $i \in D$ then $\sum_{C \ni i, C \in \mathcal{N} \backslash\{N\}} \gamma^{C}=$ $1+\gamma^{N}$, and if $i \notin D$ then $\sum_{C \ni i, C \in \mathcal{N} \backslash\{N\}} \gamma^{C}=\gamma^{N}$. Using translation invariance and positive homogeneity, inequality (27) can be rewritten as

$$
\begin{aligned}
\sum_{C \in \mathcal{N} \backslash\{N\}} \gamma^{C} v(C) & \leqslant-\rho\left(\sum_{i \in D} X_{\cdot i}+\gamma^{N} \sum_{i \in N} X_{\cdot i}\right) \\
& =-\rho(X(D))-\rho\left(\gamma^{N} X(N)\right) \\
& =-\rho(X(D))-\gamma^{N} \rho(X(N)) \\
& =v(D)+\gamma^{N} v(N),
\end{aligned}
$$

thus we have an exact game.

Proposition 4.2 shows that each exact game is generated by some risk environment with no aggregate uncertainty.

Proposition 4.2. Each game $(N, v) \in \Gamma_{\mathrm{e}}$ is induced by some risk environment $(N, S, p, X, \rho)$ such that $X$ has no aggregate uncertainty, $\Gamma_{\mathrm{e}} \subseteq \Gamma_{\text {rnau }}$.

Proof. Consider the exact game $(N, v) \in \Gamma_{\mathrm{e}}$. We specify the risk environment $(N, S, p, X, \rho)$ as follows. We introduce a state of nature for all proper non-empty subcoalitions of $N$, thus $S=2^{n}-2$. Let $p_{1}=\cdots=p_{S}=1 / S$, and let $\rho$ be the 1 -expected shortfall with $\delta=1$. For all $C \in 2^{N} \backslash\{\emptyset\}$ there exist a core element $x_{C}$ such that $x_{C}(C)=v(C)$ since $(N, v)$ is exact. Construct $X \in \mathbb{R}^{S \times n}$ as follows. We define, for all $C \in 2^{N} \backslash\{\emptyset, N\}, X_{C} .=x_{C}$. Since $x_{C}$ is a core element, it holds that $X_{C}$. $(N)=v(N)$, thus $X$ has no aggregate uncertainty. We denote the game induced by the risk environment by $\bar{v}$. Now we have for every $C \in 2^{N} \backslash\{\emptyset\}$ that

$$
\bar{v}(C)=\min _{D \in 2^{N} \backslash\{\emptyset, N\}} X_{D}(C)=v(C),
$$

thus $\bar{v}=v$.

Note that in the proof of Proposition 4.2 the sum of the entries in each row of $X$ is equal to $v(N)$. That is why we need only $2^{n}-2$ states of nature.

Combining Propositions 4.1 and 4.2 we have the following theorem.

Theorem 4.3. The class of risk allocation games with no aggregate uncertainty coincides with the class of exact games, $\Gamma_{\text {rnau }}=\Gamma_{\mathrm{e}}$.

Biswas et al. (1999) and Csóka et al. (2007a) show that a game is convex if and only if any of its subgame is exact. This result implies directly that if there are less than four players, then the class of exact games coincides with the class of convex games. Using this observation Theorem 4.3 can be reformulated as follows.

Theorem 4.4. Let $n \in \mathbb{N}$ be such that $n<4$. Then the class of risk allocation games with $n$ portfolios and no aggregate uncertainty coincides with the class of convex games with $n$ players, $\Gamma_{\text {rnau }}=\Gamma_{\mathrm{c}}$. 
Table 3

The value function of a convex game $v$.

\begin{tabular}{lr}
\hline$C$ & $v(C)$ \\
$\{1\}$ & -10 \\
$\{2\}$ & 3 \\
$\{3\}$ & -2 \\
$\{1,2\}$ & -5 \\
$\{1,3\}$ & -6 \\
$\{2,3\}$ & 2 \\
$\{1,2,3\}$ & -1 \\
\hline
\end{tabular}

Table 4

A matrix of realization vectors generating $v$.

\begin{tabular}{lllll}
\hline$S$ & $X_{.1}$ & $X_{.2}$ & $\sum_{.3}$ & $X_{s, i}$ \\
\hline 1 & -3 & 4 & -2 & 3 \\
2 & -7 & 3 & -1 & \\
3 & -10 & 5 & -1 & 4 \\
\hline
\end{tabular}

Theorem 4.4 is illustrated by the following example.

Example 4.5. In this example we show how a 3-player convex game is generated by a risk allocation game with no aggregate uncertainty. Note that the game in Table 1 of Example 3.3 is not convex since $v(\{1,2\})+v(\{1,3\})=-4-6=-10>$ $v(\{1\})+v(\{1,2,3\})=-10-1=-11$. However, by changing $v(\{1,2\})$ to -5 we get the convex game displayed in Table 3 .

This game is generated by the risk environment with matrix of realization vectors $X$ depicted in Table 4 and the risk measure of 1-expected shortfall with $\delta=1$.

Notice that the rows of $X$ correspond to appropriately chosen marginal contribution vectors. For instance, in the first row of $X$ we have the marginal contribution vector corresponding to the permutation $(3,2,1): v(\{3\})-v(\{\varnothing\})=-2-0=-2$, $v(\{2,3\})-v(\{2\})=2-(-2)=4$, and $v(\{1,2,3\})-v(\{2,3\})=-1-2=-3$. At any marginal contribution vector, there are $n$ coalitions that exactly receive their value. Thus to generate a convex game fewer states of nature are required than $2^{n}-1$. In the example 3 states of nature suffice. Also note that all rows of $X$ sum up to -1 , since the sum of the marginal contributions is always the value of the grand coalition, and there is no aggregate uncertainty.

Similarly to Proposition 3.6 we can characterize all the risk environments that generate a given exact game.

Proposition 4.6. Consider a game $(N, v) \in \Gamma_{\mathrm{e}}$. The matrix of realization vectors $X \in \mathbb{R}^{z \times n}$ without aggregate uncertainty generates $v$ if and only if $\hat{Y}(X) \in \mathcal{X}_{v}^{*}$.

Proof. Proposition 3.6 characterizes all the matrices that generate a given totally balanced game. Since by Proposition 4.1 only exact games can be generated with matrices satisfying no aggregate uncertainty, the proof is straightforward.

\section{Conclusion}

In this paper we have discussed transferable utility cooperative games derived from a risk environment: risk allocation games. We have shown that the class of risk allocation games coincides with the class of totally balanced games. This result makes sure that a regulator or performance evaluator can always allocate risk in a stable way: there will always be a core element, no matter how the risk environment is changing.

We have also studied the case when the aggregate portfolio has the same payoff in all states of nature. We proved that if there is no aggregate uncertainty then the class of risk allocation games equals the class of exact games, where for each coalition there is a core element such that the coalition gets only its stand-alone value. This means that if there is no aggregate uncertainty, then not necessarily everybody benefits from the diversification effects in a stable allocation of risk. The regulator or performance evaluator has much discretionary power in allocating risk, since for each coalition there is always a stable allocation of risk such that the coalition gets its stand-alone value.

We have characterized all the matrices of realization vectors that generate a given totally balanced game or a given exact game. In both cases the vectors derived from the matrices by juxtaposing their rows are related to the optimal solutions of a linear programming problem.

Denault (2001) shows that if a risk allocation game for an arbitrary matrix of realization vectors is convex then the risk measure by which it is induced is necessarily additive, thus the generated risk allocation game is also additive. However, by imposing some structure on the matrix of realization vectors we have proven the following theorem: If there are less than four players and the matrix of realization vectors has no aggregate uncertainty, then the generated risk allocation game is convex, and any convex game can be generated by such a risk environment. 


\section{References}

Acerbi, C., 2002. Spectral measures of risk: A coherent representation of subjective risk aversion. J. Banking Finance 26, 1505-1518.

Acerbi, C., Tasche, D., 2002. On the coherence of expected shortfall. J. Banking Finance 26, 1487-1504.

Artzner, Ph.F., Delbaen, F., Eber, J.-M., Heath, D., 1999. Coherent measures of risk. Math. Finance 9, 203-228.

Biswas, A.K., Parthasarathy, T., Potters, J.A.M., Voorneveld, M., 1999. Large cores and exactness. Games Econ. Behav. 28, 1-12.

Bondareva, Olga N., 1963. Some applications of linear programming methods to the theory of cooperative games. Problemy Kybernetiki 10, 119-139 (in Russian).

Calleja, P., Borm, P., Hendrickx, R., 2005. Multi-issue allocation situations. Europ. J. Operations Res. 164, 730-747.

Csóka, P., Herings, P.J.J., Kóczy, L.Á., 2007a. Balancedness conditions for exact games. METEOR Research Memorandum RM07/040, 1-13.

Csóka, P., Herings, P.J.J., Kóczy, L.Á., 2007b. Coherent measures of risk from a general equilibrium perspective. J. Banking Finance 31, $2517-2534$.

Denault, M., 2001. Coherent allocation of risk capital. J. Risk 4, 1-34.

Derks, J., Reijnierse, H., 1998. On the core of a collection of coalitions. Int. J. Game Theory 27, 451-459.

Dubey, P., Shapley, L.S., 1984. Totally balanced games arising from controlled programming problems. Math. Programming 29, $245-267$.

Kalai, E., Zemel, E., 1982a. Generalized network problems yielding totally balanced games. Operations Res. 30, 998-1008.

Kalai, E., Zemel, E., 1982b. Totally balanced games and games of flow. Mathematics Operations Res. 7, 476-478.

Legut, J., 1990. On totally balanced games arising from cooperation in fair division. Games Econ. Behav. 2, 47-60.

Owen, G., 1975. On the core of linear production games. Math. Programming 9, 358-370.

Predtetchinski, A., Herings, P.J.J., 2004. A necessary and sufficient condition for the non-emptiness of the core of a non-transferable utility game. J. Econ.

Theory 116, 84-92.

Schmeidler, D., 1972. Cores of exact games. J. Math. Anal. Applications 40, 214-225.

Shapley, L.S., 1967. On balanced sets and cores. Naval Res. Logist. Quart. 14, 453-460.

Shapley, L.S., 1971. Cores of convex games. Int. J. Game Theory 1, 11-26.

Shapley, L.S., Shubik, M., 1969. On market games. J. Econ. Theory 1, 9-25.

Tijs, S., Parthasarathy, T., Potters, J., Prassad, V., Rajendra, 1984. Permutation games: Another class of totally balanced games. OR Spektrum 6, 119-123. 